\title{
Improved DV-Hop Localization Algorithm based on Surround from Anchors
}

\author{
Abdel-Hamid Mohamed \\ Rashid \\ Faculty of Computers \&Informatics \\ Benha University, Egypt
}

\author{
Ashraf Hussein \\ Operation Research Center \\ Egypt
}

\author{
Diaa Salama Abd Elminaam \\ Faculty of Computers \&Informatics \\ Benha University, Egypt
}

\begin{abstract}
Advances in wireless and digital electronics have led to the invention of Wireless Sensor Network technology (WSN). The Wireless Sensor Network (WSN) is a network of tens or thousands of sensor nodes used for sensing, monitoring, and responding to events and phenomena in a specified environment. Then sent these events to places to process and analyze it and take the appropriate decisions. Many applications in Wireless Sensor Networks depend on knowing the positions of the sensor nodes. Therefore, research in localization algorithms for Wireless Sensor Networks has become an important topic. Using range-free algorithms such as the DV - hop algorithm has many drawbacks; it requires hardware support with more costs. In addition, it consumes more power. Also, it does not achieve a high rate of position accuracy. This paper presents an improvement for the traditional DV-hop algorithm. The enhanced protocol decreased the number of anchors to 5, 4 and 3 instead of using all anchors as references. This leads to enhance the position accuracy to face the needs of environmental monitoring applications. Through implementing a number of extensive simulation experiments, the enhanced algorithm outperformed the traditional DV-hop algorithm. It reduces the localization average error. That leads to improve the positioning accuracy compared with the traditional DV-hop localization algorithm.
\end{abstract}

\section{Keywords}

Wireless Sensor Networks, Localization Algorithms, DV-hop Algorithm, Localization Average Error.

\section{INTRODUCTION}

At the end of last century, scientific revolutions in the field of wireless communications and digital electronics have been raised. One of these technologies is the wireless sensor network that is denoted as WSN [1]. Usually, the WSN consists of small sized, low powered, inexpensive devices that capable of sensing and communicating wirelessly to exchange data through a gateway to end users. WSNs technology is used in numerous applications such as industrial control, observing espionage in the military sector, and environmental monitoring, etc. In addition, they are utilized to support costeffective sensing in circumstances where human intervention or wired system's deployment can be inefficient, costly, hazardous, or otherwise untenable.

From the most important applications in wireless sensor networks is object tracking. Estimating the position of an object accurately is used in many WSN such as environmental monitoring and military surveillance. The localization in WSN can be defined as the method to determine the accurate physical positions of sensor nodes. In WSNs, the sensor nodes can be classified into two types: anchor nodes and unknown nodes. The localization in WSN can be utilized in two fields: firstly; the gathered data are of no importance if the locations of the sensors are not known, especially in the environmental monitoring applications and tracking the targets. Secondly; routing techniques that dependent on the position information [2].

Most researches in the WSNs classify the localization algorithms into two main classes: range-based [3] and rangefree [4]. Range-based methods use distance or angle estimates in their locations computations, while range-free techniques are based on the connectivity information between unknown nodes and anchors. Range-based techniques have used Received Time of Arrival (TOA) [3], Signal Strength (RSS) [5], Time Difference of Arrival (TDoA) [6], or Angle of Arrival (AoA) [7]. Anchor nodes can get its own coordinates information in advance by GPS technology or the artificial deployment information. In this paper, Unknown node estimates its own position based on connectivity to 5,4 or 3 anchors instead using all the anchors in the network.

The paper structure is organized as follows: section 2 quickly introduces the classification of WSN localization algorithms, in section 3; proposed enhanced algorithm is described in details, in section 4; experiments and simulation results of the proposed improved algorithm compared to the traditional DVhop algorithm and in Section 5; conclusion.

\section{CLASSIFICATION OF LOCALIZATION ALGORITHMS}

Generally, the GPS devices [8] are not used in large-scale sensor network due to the cost and the weaken signals inside the indoor environments. As shown in fig 1 the localization algorithms for WSNs can be classified into two main categories: range-based and range-free. Range-based schemes use range measurements between sensor nodes to estimate the coordinates of unknown nodes, whereas range-free approaches do not require this data.

\subsection{Range-based techniques}

- The Time of Arrival (ToA) [3]: after registering the transmission time $T 0$ of sending node and the reception time $T 1$ of the receiving node, the distance between the 2 nodes is computed by multiplying the signal propagation speed and the time distinction. The technique is loaded on the hardware devices so; it is expensive because each node needs strict time synchronization; thus, it is not appropriate for use in indoor positioning.

- The Time Difference of Arrival (TDoA) [6] is the variance in arrival time between signals received separated receivers; in common, it is used for source localization measurements. 


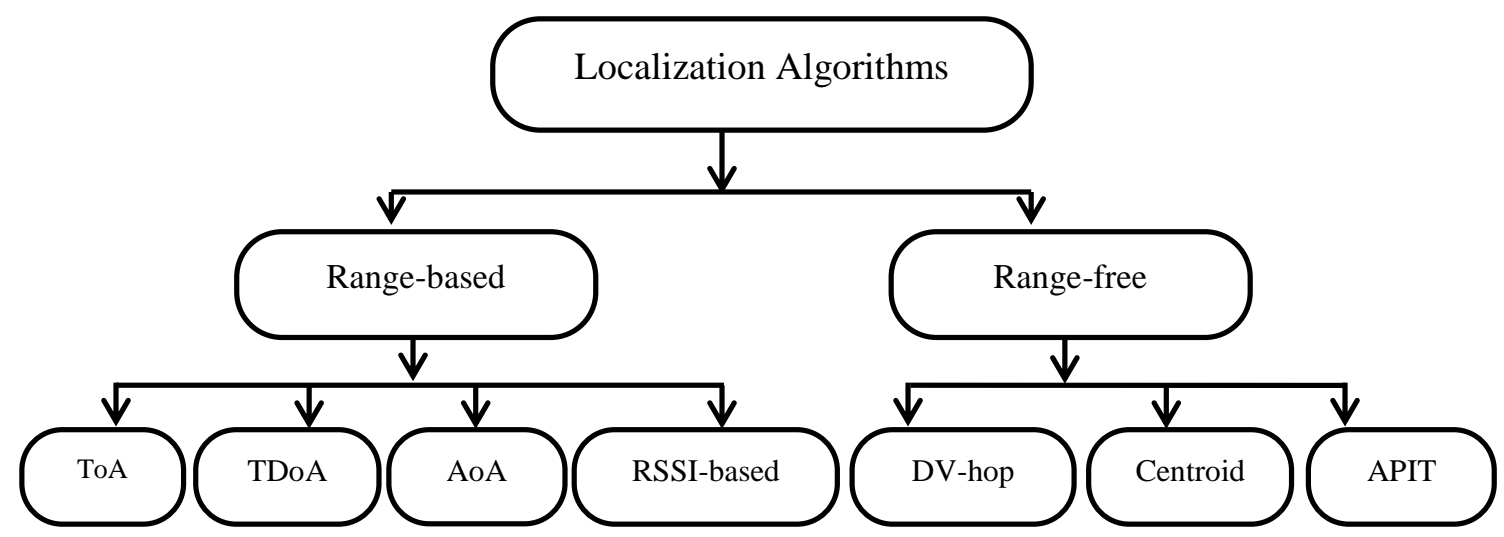

Fig 1: Localization Algorithms classification

- $\quad$ The Angle of Arrival (AoA) [7] based localization is a passive localization technique whereby the location of an emitter is getten by triangulation of bearing information collected at a number of sensors. Each node is equipped with an antenna array angle measuring device to determine the direction of the node, so this algorithm additionally requires all the more high performance hardware.

- RSSI-based algorithm [5] uses of the shadowing transmission model to compute the distance between the anchor node (known node) and the blind node (unknown node) and then calculate the coordinate by trilateration method. It has many advantages, including less requirements of hardware and is very simple and cheap. Therefore, it becomes one of the most widely used indoor localization algorithms. However, it may be irregular signal propagation characteristics (fading, interference, multi-path etc.)

\subsection{Range-free technique}

Do not use any ranging at all, simply deploy enough anchors. Anchors periodically broadcast their location. Good anchor placement is crucial.

Example: DV-hop algorithm [9], centroid algorithm [10], and APIT algorithm [7].

\subsection{DV-hop Algorithm}

DV-hop algorithm is the most famous algorithm within Range-free technique. It is widely used now, for the reason that it is not complex, and is more practical. There are researches propose enhancements to correct the position of DV-hop based on the estimated distance to the nearest anchors.

Step 1: the algorithm starts when every anchor node floods its information (id, coordinates, hop number), all nodes in the range (anchors, blind nodes) can obtain this information.

Step 2: every anchor node calculates the average distance per hop (average size per one hop) to other anchors from the following formula. Then, the calculated average size is broadcasted to every node entire the network.

hopsize $_{i}=\frac{\sum_{j \neq i} \sqrt{\left(x_{i}-x_{j}\right)^{2}+\left(y_{i}-y_{j}\right)^{2}}}{\sum_{j \neq i} h i j}$

The parameters (xi, yi), (xj, yj) are the coordinates of anchor $\mathrm{i}$ and anchor $\mathrm{j}$, where hij are the hops between anchor $\mathrm{i}$ and anchor $\mathrm{j}$.
Step 3: each unknown node collects values of the hop size from anchor nodes which have the least hops between them, it calculates the distance to the anchor nodes from the following formula.

$\mathrm{d}_{i}=\mathrm{h}_{i d} *$ hopsize $_{i}$

Where $\mathrm{h}_{\mathrm{id}}$ is the minimum hop count from the unknown node to the anchor node. Then, every unknown node fixes its closest anchor, which will be considered as a reference node.

Step 4: every unknown node calculates its coordinates based on all anchor nodes in the network. It can be obtained from the following formulas.

To get the coordinates of $\mathrm{X}$ blind node where the coordinates of $\mathrm{X}$ are presented as an array in the following equation.

$X=\left[\begin{array}{l}x \\ y\end{array}\right]$

Where $X=\left(A^{T} A\right)^{-1} A^{T} B$

Where $\mathrm{A}$ and $\mathrm{B}$ are

$A=\left[\begin{array}{cc}2\left(x_{1}-x_{n}\right) & 2\left(y_{1}-y_{n}\right) \\ 2\left(x_{2}-x_{n}\right) & 2\left(x_{2}-x_{n}\right) \\ \cdots & \\ 2\left(x_{n-1}-x_{n}\right) & 2\left(x_{n-1}-x_{n}\right)\end{array}\right]$,

$B=\left[\begin{array}{c}d_{1}^{2}-d_{n}^{2}-x_{1}^{2}+x_{n}^{2}-y_{1}^{2}+y_{n}^{2} \\ d_{2}^{2}-d_{n}^{2}-x_{2}^{2}+x_{n}^{2}-y_{2}^{2}+y_{n}^{2} \\ \vdots \\ d_{n-1}^{2}-d_{n}^{2}-x_{n-1}^{2}+x_{n}^{2}-y_{n-1}^{2}+y_{n}^{2}\end{array}\right]$

\section{THE PROPOSED OPTIMIZATION IN DV-HOP ALGORITHM}

The objective of using the optimized localization algorithm is estimating the locations of the blind nodes indoor or outdoor depending on the known data from least number of the anchor nodes. The proposed optimization of the DV-hop algorithm is discussed in details in this section. Firstly, subsection 3.1 discusses an assessment method to measure the accuracy of the proposed algorithm

\subsection{Localization accuracy}

There are two assessment methods to measure the correct rate out the accuracy of the localization algorithm. First one is the distance error and the second is the localization average error.

The distance error for every unknown node entire the network $\left(\right.$ Error $\left._{\mathbf{n}}\right)$ is the distance between the estimated 
coordinate and the actual coordinate. It is calculated using the following equation [9]:

$\operatorname{Error}_{n}=\sqrt{\left(x_{n}^{\prime}-x_{n}\right)^{2}+\left(y_{n}^{\prime}-y_{n}\right)^{2}}$

Where $\left(\boldsymbol{x}_{\boldsymbol{n}}^{\prime}, \boldsymbol{y}_{\boldsymbol{n}}^{\prime}\right)$ are the estimated coordinates of node N and $\left(\mathbf{x}_{\mathbf{n}}, \mathbf{y}_{\mathbf{n}}\right)$ are the actual coordinates of the same node.

The localization average error (AveError) is the most important factor of the WSN localization. It can be expressed by the following equation [9]:

AveError $=\frac{1}{\mathrm{NR}} * \sum_{\mathrm{n}=1}^{\mathrm{N}}$ Error $_{n} * 100 \%$

The parameter $\mathbf{N}$ is the number of nodes and $\mathbf{R}$ is the communication radius of the sensor node.

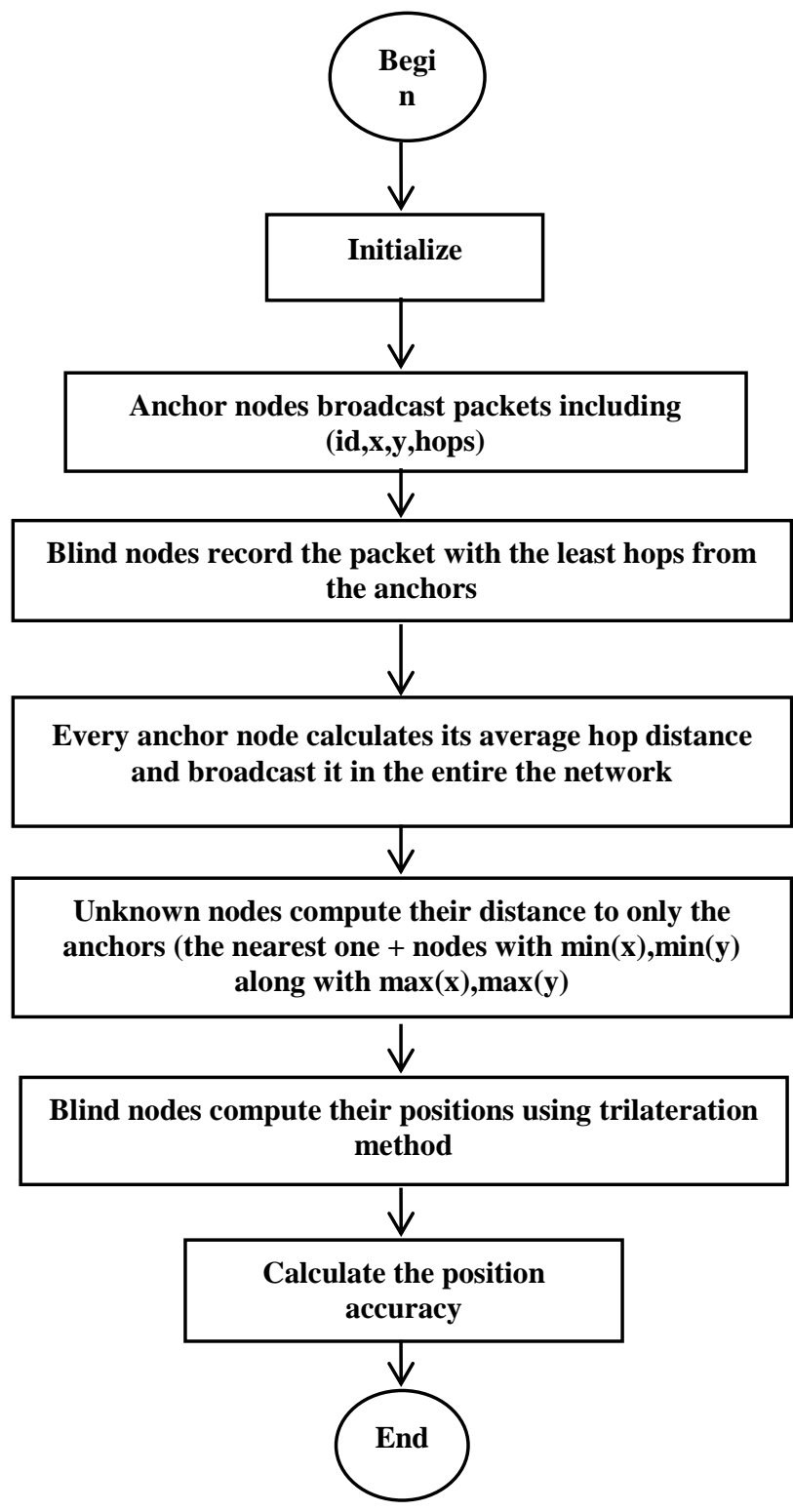

Fig 2: The improved DV-hop algorithm

\subsection{Description of the improved DV-hop}

The accuracy of the proposed algorithm outperforms the accuracy of the DV-hop algorithm where it reduces the distance error for the most nodes. The proposed algorithm considers the anchors with the highest $\mathrm{X}$-coordinate, the highest Y-coordinate along with anchors with lowest Xcoordinate, and lowest Y-coordinate to establish a virtual square around those blind nodes. Thus, the selective anchors may be 4 or 3 or 2 anchors based on the deployment. Therefore, it is better to increase the number of references by adding the nearest anchor to allow using of the mathematical trilateration method.

In contrast, the original DV-hop increases the network overhead because it uses all anchor nodes to estimate the location [9]. There are other improvements in the DV-hop algorithm as in [11], but it is less overhead, it selects one nearest anchor and any two anchors to estimate the location, but the drawback of this algorithm is the deployment of the three anchors may be in one side angle from the blind node that causes high distance error.

The flowchart in fig 2 models the proposed improvement of DV-hop algorithm. The following steps describe in detail the proposed algorithm:

Step 1: the algorithm starts when each anchor node broadcasts its information (id, coordinates, and hops). All nodes in the range (anchors, blind nodes) can obtain this information and update the initial value of hop number from 0 to be 1 . The hops from any blind node to any anchor node are obtained. In addition, the anchor node accesses to the coordinates and hops of all other anchor nodes.

Step 2: every anchor node computes the average distance per hop (average size per one hop) of the entire network to other anchors from the equation 1 . Then, the estimated average size is broadcasted to every node in the whole network.

Step 3: every unknown node receives the hop size from anchors, which have the least hops between them; it calculates the distance to the anchors which have the highest $\mathrm{X}$ and $\mathrm{Y}$ coordinate and lowest $\mathrm{X}$ and $\mathrm{Y}$-coordinate. These anchors may be less than 4 anchors; also, the nearest anchor is selected. The distance is obtained from equation 2 .

Step 4: each blind node calculates its coordinates based on 5 anchors or less to 3 anchors; it can be obtained from the following equation 8 .

$$
\begin{aligned}
& \left(x-x_{1}\right)^{2}+\left(y-y_{1}\right)^{2}=d_{1}^{2} \\
& \left(x-x_{2}\right)^{2}+\left(y-y_{2}\right)^{2}=d_{2}^{2} \\
& \left(x-x_{n}\right)^{2}+\left(y-y_{n}\right)^{2}=d_{n}^{2}
\end{aligned}
$$

\section{Where $\mathrm{n}=5$ or 4 or 3}

To get the coordinates of $\mathrm{X}$ blind node where the coordinates of $\mathrm{X}$ is presented as an array in equation 3 and equation 4 respectively.

Where $\mathrm{A}$ and $\mathrm{B}$ are:

$A=\left[\begin{array}{cc}2\left(x_{1}-x_{n}\right) & 2\left(y_{1}-y_{n}\right) \\ 2\left(x_{2}-x_{n}\right) & 2\left(x_{2}-x_{n}\right) \\ \cdots & \\ 2\left(x_{n-1}-x_{n}\right) & 2\left(x_{n-1}-x_{n}\right)\end{array}\right]$, 
$B=\left[\begin{array}{c}d_{1}^{2}-d_{n}^{2}-x_{1}^{2}+x_{n}^{2}-y_{1}^{2}+y_{n}^{2} \\ d_{2}^{2}-d_{n}^{2}-x_{2}^{2}+x_{n}^{2}-y_{2}^{2}+y_{n}^{2} \\ \vdots \\ d_{n-1}^{2}-d_{n}^{2}-x_{n-1}^{2}+x_{n}^{2}-y_{n-1}^{2}+y_{n}^{2}\end{array}\right]$

Where $\mathrm{n}=5$ or 4 or 3

\subsection{Walk through the proposed algorithm}

Fig 3 presents network from 6 anchors and one unknown node. The goal from this example is to estimate the coordinates of the blind node using the above steps of the improved algorithm.

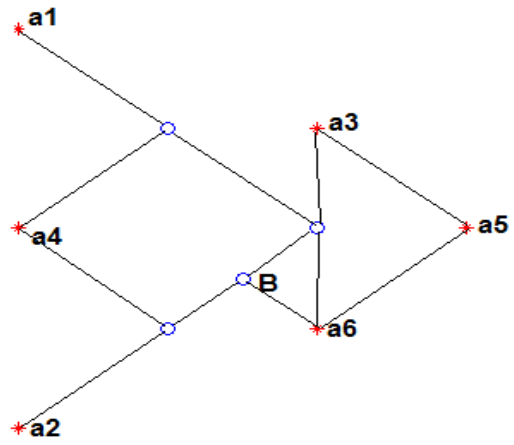

Fig 3: Explained example

Assume a1, a2,a3 ,.., a6 are 6 anchor nodes. Node B is the unknown node which needs to be located. The proposed algorithm works as follows.

Firstly, anchor nodes broadcast packets including their $\mathrm{x}, \mathrm{y}$ coordinates and a flag which is initialized as 0 to calculate the number of hops away from the other nodes. When the packet is forwarded, the hop number is increased by 1 . Therefore, each blind node will know the hop distances from itself to all anchors.

Table 1. Node B table example

\begin{tabular}{|c|c|c|c|}
\cline { 2 - 4 } \multicolumn{1}{c|}{} & Hops & \multicolumn{2}{c|}{$\mathbf{x , y}$} \\
\hline $\mathbf{a 1}$ & 3 & 10 & 50 \\
\hline $\mathbf{a 2}$ & 2 & 10 & 10 \\
\hline $\mathbf{a 3}$ & 2 & 30 & 40 \\
\hline $\mathbf{a 4}$ & 2 & 10 & 30 \\
\hline $\mathbf{a 5}$ & 2 & 40 & 30 \\
\hline $\mathbf{a 6}$ & 1 & 30 & 20 \\
\hline
\end{tabular}

Each anchor will calculate the average distance per hop after receiving the beacon messages as follows:

$\mathrm{a} 1=(40+22.3+20+36+31.6) /(5+2+2+3+3)=10$; $\mathrm{a} 2=(40+36+20+36+22.5) /(5+4+2+3+3)=12$

Also hop size of $\mathrm{a} 3$ and $\mathrm{a} 4=10.4 ; \mathrm{a} 5=11.8$ and $\mathrm{a} 6=11$.

After calculating the average distance per hop, the anchor nodes will broadcast the value through the network. Then each blind node receives the average distance per hop from the anchors.

It calculates the distance to only the anchors: nearest anchor node and anchors with the highest $(\mathrm{x}, \mathrm{y})$ and lowest $(\mathrm{x}, \mathrm{y})$ coordinates. Blind node $\mathrm{B}$ will select the anchors a1, a2, a5 and a6 to calculate its distance to them by using the equations 3.4 as follows:

Table2. Node B update table to add hop-size

\begin{tabular}{|c|c|c|c|c|}
\cline { 2 - 5 } \multicolumn{1}{c|}{} & Hops & \multicolumn{2}{c|}{$\mathbf{x , y}$} & Hops-size \\
\hline $\mathbf{a 1}$ & 3 & 10 & 50 & 10 \\
\hline $\mathbf{a 2}$ & 2 & 10 & 10 & 12 \\
\hline $\mathbf{a 3}$ & 2 & 30 & 40 & 10.4 \\
\hline $\mathbf{a 4}$ & 2 & 10 & 30 & 10.4 \\
\hline $\mathbf{a 5}$ & 2 & 40 & 30 & 11.8 \\
\hline $\mathbf{a 6}$ & 1 & 30 & 20 & 11 \\
\hline
\end{tabular}

$\mathrm{d} 1=10 * 3=30 ; \mathrm{d} 2=12 * 2=24 ; \mathrm{d} 3=11.8 * 2=23.6 ; \mathrm{d} 4=11$

Table 3. Node B distance table

\begin{tabular}{|c|c|}
\cline { 2 - 2 } \multicolumn{1}{c|}{} & Distance \\
\hline d1 & 30 \\
\hline d2 & 24 \\
\hline d3 & 23.6 \\
\hline d4 & 11 \\
\hline
\end{tabular}

The blind node $\mathrm{B}$ will calculate its coordinates based on 2 anchors a1, a3; it can be obtained from the following linear equations.

$$
\begin{aligned}
& (x-10)^{2}+(y-50)^{2}=900 \\
& (x-10)^{2}+(y-10)^{2}=576 \\
& (x-40)^{2}+(y-30)^{2}=556.9 \\
& (x-30)^{2}+(y-20)^{2}=121
\end{aligned}
$$

The above equations can be represented in matrix $\mathrm{A}$ and matrix $\mathrm{B}$, then using last equation 9 to find the coordinates of $\mathrm{B}$ that will be (29 and 23.8). Notice that the actual coordinates of B node are (25 and 25) but when the DV-hop is applied in this example the computed coordinates are (31.1 and 22.3). Also; by testing the selective 3-anchors DV-hop algorithm [11] the localization average results are (31 and 15.7).

\section{PERFORMANCE EVALUATION OF THE IMPROVED DV-HOP ALGORITHM}

Evaluating the improved DV-hop performance is carried out using Matlab 7.8.0 by simulating the network scenarios and determines the localization error results. The paper supposes that anchor nodes are deployed randomly in a 2-dimensional area. The area is $100 * 100$ square meters. The communication radius of the anchor nodes and blind nodes is 20 meters. Nodes distribution is shown in fig 4. The anchor nodes are blue stars, the unknown nodes are black points, and the anchors that selected to be referenced are blue star in a red circles.

It is important to compute the localization average error of the proposed improved DV-hop algorithm to evaluate its accuracy. The actual coordinates are compared to the estimated nodes to examine the position accuracy of the improved localization algorithm. Three scenarios are 
proposed to evaluate the performance of the improved algorithm compared to the original DV-hop algorithm performance:

- The distance error per every blind node.

- The impact of using a different density of anchors.

- The impact of using different communication radius.

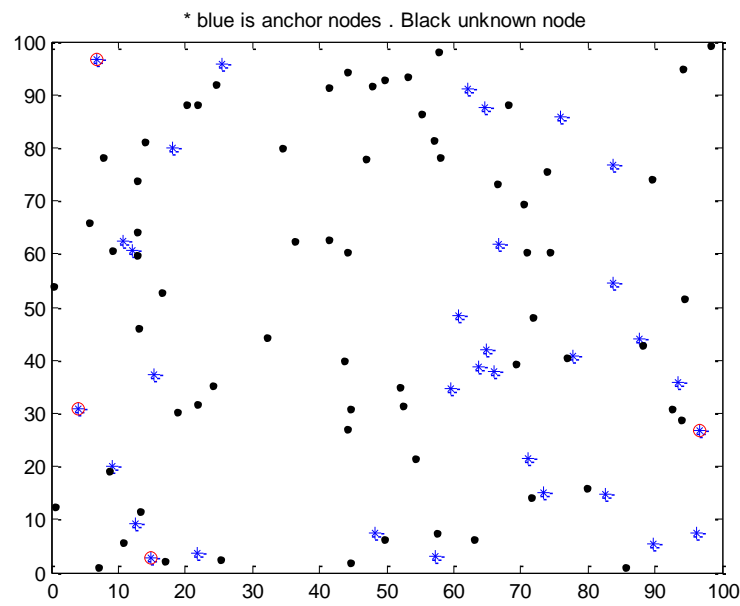

Fig 4: The random distribution of the nodes

\subsection{The distance error of blind nodes}

Through this experiment, the distance error for every node is calculated. The parameters of the anchor nodes ratio are fixed at $9 \%$. Also, the communication range is fixed at $20 \mathrm{~m}$. Fig 5 represents a comparison between the distance errors for every blind node at the traditional DV-hop algorithm and the improved DV-hop algorithm. It is clear that the distance errors calculated by the improved algorithm are smaller than those calculated by the traditional DV-hop algorithm.

Blue is traditional DV-hop, Red is improved DV-hop

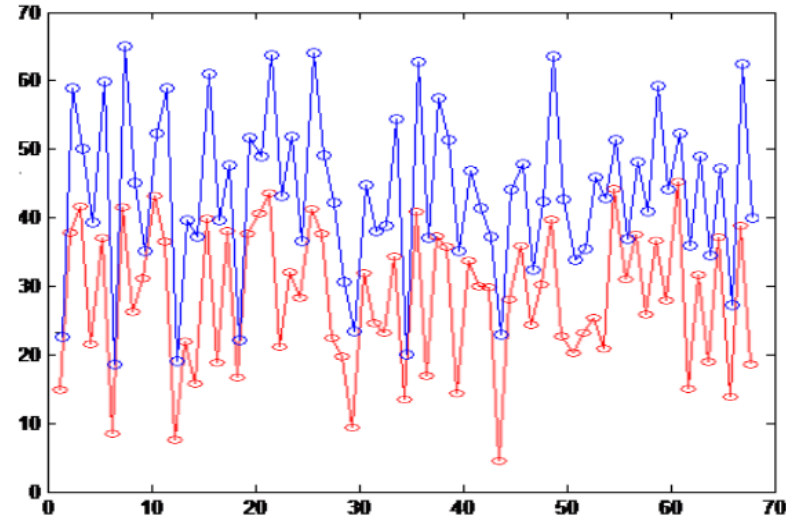

Fig 5: Distance error per node

\subsection{The Impact of using different anchors densities}

The position accuracy of the improved DV-hop algorithm is affected by the factor of the anchor nodes ratio. The higher ratio of the anchor nodes generates higher position accuracy. In this simulated experiment the total number of nodes is fixed to 200 , communication range is fixed to $20 \mathrm{~m}$, and the ratio of anchor nodes is increased from $10 \%$ to $50 \%$ to observe its influence on the algorithm. Fig 6 represents the impact of using different densities of anchor nodes. The proposed improved algorithm optimizes the position accuracy over the traditional DV-hop in the case of different anchor node ratios, and in a very small proportion of anchor nodes (above 9\%); the position accuracy is about $8 \%$ higher than that of the traditional DV-hop. With the increase of the ratio of anchor nodes, the position accuracy of the proposed improved algorithm is also improved.

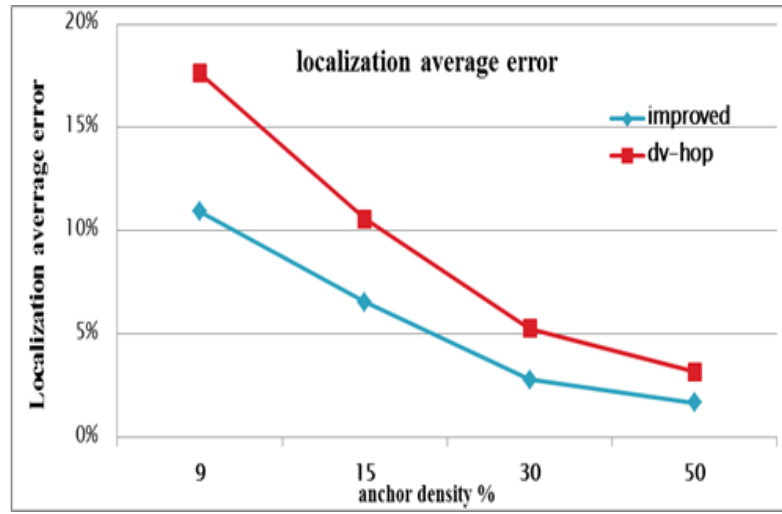

Fig 6: Localization average error under different anchor densities

\subsection{The impact of communication radius}

The communication radius of the sensor node affects the position accuracy of the localization algorithm. In this simulated experiment, the anchor nodes density are fixed at $15 \%$ and increase the total number of the nodes to 200 nodes. The communication radius is changed from $20 \mathrm{~m}$ to $80 \mathrm{~m}$. Fig 7 shows that the improved algorithm optimizes the accuracy over the traditional DV-hop in the all of different communication radius values. When communication radius is $20 \mathrm{~m}$, the position accuracy of the improved algorithm is about $5 \%$ higher than that of DV-hop. With the increase of the communication radius, the position accuracy of the proposed algorithm is also improved.

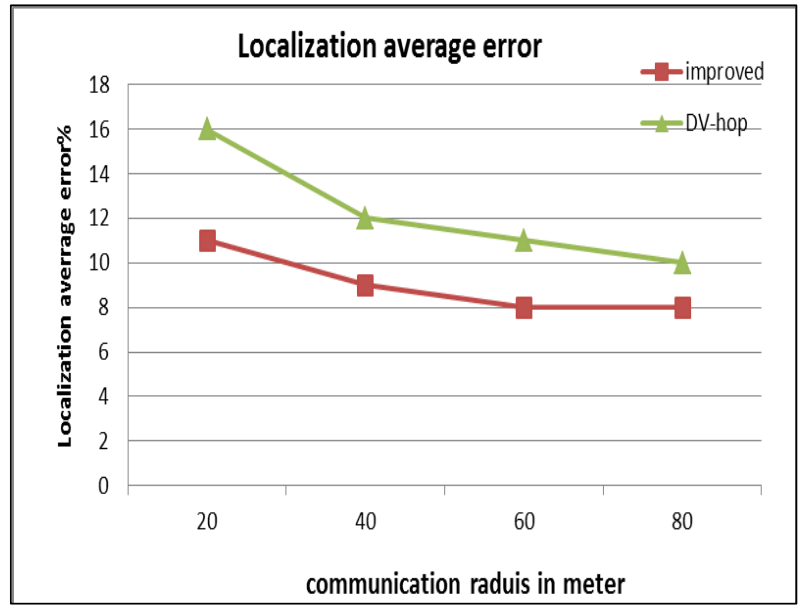

Fig 7: Average location error under different communication radius

\section{CONCLUSION}

The research paper presents an improved version of the traditional DV-hop algorithm; the proposed algorithm reduces the distance error for each blind node and overall average distance. Thus, it improves the accuracy. Also; it reduces the network overhead by filtering the type of selective anchors based on the parameters of (anchors with highest $\mathrm{x}$ and $\mathrm{y}$ coordinates) along with (the anchors with lowest $\mathrm{x}$ and $\mathrm{y}$ 
coordinates). Also, the proposed algorithm decreases the number of anchors is 5, 4 and 3 instead of using all anchors as references. Through a number of performed simulation experiments, the enhanced algorithm improves the localization average.

\section{REFERENCES}

[1] I.F. Akyildiz, W. Su, Y. Sankarasubramaniam, and E. Cayirci, "Wireless Sensor Networks: A Survey", IEEE Computer Networks, Vol. 38, No. 4,March 2002, pp. $393-422$.

[2] Jeril Kuriakose, Sandeep Joshi and V.I. George," Localization in Wireless Sensor Networks: A Survey"; CSIR Sponsored X Control Instrumentation System Conference - CISCON-2013.

[3] G.L, E.D, "Robust Range Estimation Using Acoustic And N Multimodal Sensing" IEEE International Conference on Intelligent Robots and Systems, Vol 3, pp. 1312-1320, 2001.

[4] T. He, C. Huang, B. M. Blum, J. A. Stankovic, T.Abdelzaher."Range-Free Localization Schemes For Large Scale Sensor Networks" Proc. of the ACM MobiCom 2003, San Diego, pp. 81-95, 2003.

[5] E.Goldoni, A. Savioli, M. Risi, and P. Gamba, "Experimental Analysis Of RSSI-Based Indoor Localization With IEEE 802.15.4" in Wireless Conference (EW), 2010 European, 2010, pp. 71-77.
[6] X.Cheng, T. A, G. Xue, D. Chen, "TPS: Time-Based Positioning Scheme for Outdoor Wireless Sensor Networks" IEEE INFOCOM2004, Hong Kong, China. pp. 2685-2696, March, 2004.

[7] T. D. J. "Statistical theory of Passive Location Systems," IEEE Trans. on AES,Vol.20, No.2, pp. 183-198, Mar, 1984

[8] L. Peneda, A. Azenha, and A. Carvalho, "Trilateration for Indoors Positioning Within The Framework Of Wireless Communications," in Industrial Electronics, 2009. IECON '09. 35th Annual Conference of IEEE, 2009, pp. 2732-2737.

[9] D. Niculescu, B. Nath. "Ad Hoc Positioning System (APS)," Proc. of the IEEE GLOBECOM 2001, San Antonio, pp. 2926-2931, 2001.

[10] C.S, H. M, H.J, "GPS-Free Positioning in Mobile Ad Hoc networks" in Proc. of Hawaii Int'l. Conf. System Sciences, pp.3481-3490, 2001.

[11] Hichem Sassi, Tawfik Najeh, Noureddine Liouane, "A Selective 3-Anchor DV-Hop Algorithm Based On the Nearest Anchor for Wireless Sensor Network", International Journal of Computer, Electrical, Automation, Control and Information Engineering $\mathrm{Vol}$ 8, No. 10, pp. 2014. 